\title{
Polinizadores visitam o girassol durante todo florescimento, aumentando a produtividade e uniformizando as sementes
}

\section{Pollinators visit the sunflower throughout flowering, increasing yield and standardizing the seeds}

Rafael Kill-Silveira - Doutorando no Programa de Pós-graduação em Ciências Ambientais da Universidade do Estado de Mato Grosso (UNEMAT). E-mail: killsilveira.r@gmail.com

Luiz Fernando Rodriguês Cordeiro - Graduado em Zootecnia pela Universidade do Estado de Mato Grosso (UNEMAT). E-mail: luizfernando_foi@hotmail.com

Dionei José da Silva - Doutor em Biologia Tropical e Recursos Naturais pelo Instituto Nacional de Pesquisas da Amazônia (INPA). Professor adjunto da Universidade do Estado de Mato Grosso (UNEMAT). E-mail: dioneijs@gmail.com

\section{Resumo}

Objetivou-se com esta pesquisa verificar o efeito da polinização biótica na cultura do girassol, associada ou não à adubação orgânica, e ainda determinar em qual período de florescimento e qual horário do dia há maior abundância de visitantes florais, na tentativa de informar em qual momento é seguro o uso de substâncias nocivas aos polinizadores. Foram utilizadas 52 plantas em delineamento inteiramente casualizado com arranjo fatorial 2x2 (adubação x polinização biótica). Os visitantes florais foram observados das 07:00 às 18:00 horas durante 1 minuto a cada hora. Houve incremento produtivo de até $25 \%$ com a polinização biótica no girassol, mas este efeito é dependente do conteúdo de nutrientes disponíveis às plantas. Houve presença de polinizadores durante todo o período de florescimento e em qualquer horário do dia, desta maneira, só é viável economicamente o uso de agrotóxicos se as perdas de produção ocasionada pelas pragas forem superiores a $25 \%$.

\section{Palavras-chave}

Abelhas nativas. Adubação orgânica. Agroecossistemas. Helianthus annuus. Polinização. Serviços ecossistêmicos.

\begin{abstract}
We aim with this research to verify the effect of the biotic pollination on sunflower culture associated or not with organic fertilization, and also to determine in which flowering period and what time of the day there is a greater abundance of floral visitors, in an attempt to inform at what time the use of substances harmful to pollinators is safe. 52 plants were used in a completely randomized design with a $2 \times 2$ factorial arrangement (Fertilization $\mathrm{x}$ biotic pollination). Floral visitors were observed from 7:00 am to 6:00 pm for 1 minute every hour. There was a productive increase of up to $25 \%$ with biotic pollination in sunflower, but this effect is dependent on the content of nutrients available to the plant. Pollinators were present during all the flowering period and at any time of the day, so the use of pesticides is only economically viable if the production losses caused by the pests are greater than $25 \%$.
\end{abstract}

\section{Keywords}

Agroecosystems. Ecosystemic services. Helianthus annuus. Organic fertilizer. Pollination. Wild bees. 


\section{INTRODUÇÃO}

O Brasil se comprometeu perante o Programa das Nações Unidas para o Desenvolvimento, a cumprir com as 169 metas dos 17 Objetivos do Desenvolvimento Sustentável (ODS). Dentre outros, destaca-se o objetivo 2, que é o de acabar com a fome, alcançar a segurança alimentar e melhoria da nutrição, promovendo a agricultura sustentável até o ano de 2030. A alimentação humana é mantida em quase sua totalidade pela agricultura, no entanto, da forma como vem sendo praticada está entre as principais fontes de degradação do meio ambiente (CLARK; TILMAN, 2017). Um dos maiores desafios é garantir a alta produtividade agrícola associada à conservação ambiental (PHALAN et al., 2016).

Segundo Bommarco, Kleijn e Potts (2013), a intensificação ecológica é uma estratégia viável para a minimização dos danos causados pela agricultura moderna, e que simultaneamente, contribui para o aumento da produção agrícola. A intensificação dos serviços ecossistêmicos de polinização é um dos exemplos. De acordo com Klein et al. (2007), mais de 70\% das culturas agrícolas e 35\% da produção agrícola dependem de insetos polinizadores.

Globalmente o serviço ecossistêmico de polinização foi estimado em 173 bilhões de dólares ao ano em 2009 (GALLAI; VAISSIÈRE, 2009). Para o Brasil, este valor foi de $\mathrm{R} \$ 31$ bilhões em 2016, o impacto negativo da perda de polinizadores será maior para as culturas mais dependentes de polinização, como exemplo a cultura do girassol (Helianthus annuus L.), que em 2009 a produção rendeu para o país $\mathrm{R} \$ 85$ milhões, e os serviços de polinização corresponderam a R\$ 55 milhões, entretanto a eficácia deste serviço pode estar ameaçada, pois o uso de agrotóxicos no Brasil aumentou 278\% entre os anos de 2002 a 2016 (SILVEIRA; GALBIATI, 2019).

Sobretudo, porque de acordo com Jung (2014), a redução da população de polinizadores associa-se principalmente ao uso indiscriminado de agrotóxicos. $\mathrm{E}$ ainda, segundo Oliveira et al. (2018), o uso de agrotóxicos diminui a abundância de polinizadores e, como consequência, a produtividade do girassol. Acreditavase que apenas os inseticidas eram os responsáveis pela morte de abelhas, contudo, são vários os ingredientes ativos (IAs) de agrotóxicos que causam malefícios a estes seres vivos (BOILY et al., 2013; DEGRANDI-HOFFMAN et al., 2015; IUPAC, 2018; KIDD; JAMES, 1991; LEITE et al., 2015).

As abelhas são os principais polinizadores, e dentre estas, a espécie Apis mellifera é a mais utilizada, porém, para a cultura do girassol tem sido descrito que as espécies nativas são mais eficientes no processo de polinização (GREENLEF; KREMEN, 2006; MALLINGER et al., 2019). As doses letais de agrotóxicos para 
algumas espécies de abelhas nativas são inferiores quando comparados com as doses letais para a espécie Apis mellifera, que é a espécie utilizada nos testes de toxicidade (ARENA; SGOLASTRA, 2014; VALDOVINOS-NÚÑEZ et al., 2009).

Para Perrot et al. (2018), o rendimento de culturas agrícolas como o girassol poderia ser aumentado por meio do manejo de colmeias de abelhas e da densidade de plantas, sem recorrer a agroquímicos. Sendo assim existem estratégias de manejo ainda não utilizadas por técnicos e produtores que podem contribuir para diminuir os danos causados, aumentando a produtividade e lucratividade dos sistemas agrícolas. Concomitantemente, os benefícios da polinização biótica dependem do genótipo de girassol utilizado para o cultivo (MALLINGER; PRASIFKA, 2017a).

Objetivou-se com esta pesquisa verificar o efeito da polinização biótica na cultura da cultivar de girassol SYN 039A, associada ou não à adubação orgânica e, ainda, determinar em qual período de florescimento e qual horário do dia há maior abundância de visitantes florais, na tentativa de informar aos técnicos e produtores em que momento é seguro o uso de substâncias nocivas aos polinizadores.

\section{MATERIAL E MÉTODOS}

O experimento foi conduzido no setor de Apicultura da Universidade do Estado de Mato Grosso, câmpus Universitário de Pontes e Lacerda, o qual está localizado sob as coordenadas geográficas 15³2'66.3” Sul e 59²3'24.4" Oeste, com altitude média de $254 \mathrm{~m}$ acima do nível do mar. Segundo Koppen (1923), o clima da região é de classificação do tipo Aw, com chuvas concentradas no verão (outubro a abril), e com período seco bem definido durante a estação de inverno (maio a setembro), e apresenta precipitação média anual de $1.500 \mathrm{~mm}$, com temperatura média de $24^{\circ} \mathrm{C}$, a máxima de $38^{\circ} \mathrm{C}$ e a mínima $14^{\circ} \mathrm{C}$. O solo é classificado como Argissolo Vermelho distrófico, de textura médio arenosa.

Foram utilizadas sementes do girassol de genótipo SYN 039A. O plantio foi realizado em copos descartáveis de $50 \mathrm{~mL}$ na profundidade de semeadura de $3 \mathrm{~cm}$ em agosto de 2016. Quinze dias após a germinação as plantas foram transferidas para vasos de plástico com 32x23x19 cm de diâmetro superior, inferior, e altura, respectivamente. Os vasos foram alocados com espaçamento de $70 \mathrm{~cm}$ entre linhas em casa de vegetação. Para eliminar o efeito da localização os vasos foram dispostos de maneira intercalada (um com adubo e outro sem adubo).

O substrato utilizado nos vasos foi solo e esterco de poedeiras. A quantidade de fertilizante utilizada foi de $20 \mathrm{Mg}$ ha- $^{1}$ (ALVAREZ et al., 2006). 
Para o cálculo da quantidade de adubo em cada vaso foi considerado que o solo apresentava a densidade de $1,2 \mathrm{~g} \mathrm{dm}^{3}$. Tendo como base a profundidade de 20 $\mathrm{cm}$, teremos 2.400.000,00 kg ha-1 de solo. Dessa forma cada vaso recebeu $11 \mathrm{~kg}$ de solo e $90 \mathrm{~g}$ de esterco.

A adubação foi dividida em três doses (30 g cada), sendo que a primeira ocorreu no momento da transferência das plântulas para o vaso, a segunda aos 30, e a terceira aos 40 dias após a semeadura. Anteriormente, a adubação, e de acordo com a análise física, o solo apresentava os teores de argila, silte e areia, nas concentrações de 186,8, 776,0, 735,6 g kg-1, respectivamente. Os respectivos teores da composição química podem ser observados na Tabela 1. As análises foram realizadas no laboratório de análise de solos da Universidade do Estado de Mato Grosso, campus universitário de Pontes e Lacerda.

Tabela 1 - Composição química do solo utilizado

\begin{tabular}{|c|c|c|c|c|c|c|c|c|c|c|c|c|c|c|}
\hline $\begin{array}{l}0_{N} \\
\mathbb{I}^{\prime} \\
\mathbb{Z}_{2}\end{array}$ & 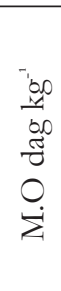 & $\begin{array}{l}\hat{E} \\
\tilde{E} \\
0 \\
\tilde{E} \\
\tilde{c}\end{array}$ & $\begin{array}{l}\tilde{E} \\
\tilde{E} \\
60 \\
\Xi \\
\underline{V}\end{array}$ & 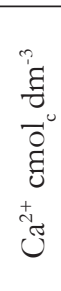 & 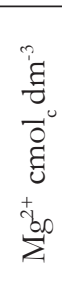 & 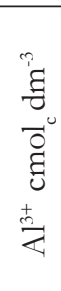 & 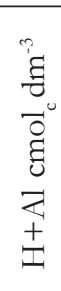 & 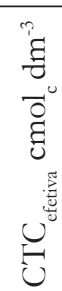 & 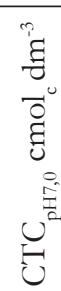 & $\begin{array}{l}0^{\circ} \\
>\end{array}$ & 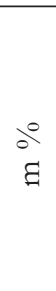 & $\begin{array}{l}{ }^{\circ} \\
\ddot{L}\end{array}$ & $\begin{array}{l}0^{\circ} \\
\tilde{U}\end{array}$ & $\begin{array}{l}0^{\circ} \\
\sum^{\infty}\end{array}$ \\
\hline 6,0 & 1,5 & 6,0 & 92,6 & 2,2 & 1,0 & 0,0 & 2,6 & 3,4 & 6,0 & 57,3 & 0 & 7 & 64,7 & 29,4 \\
\hline
\end{tabular}

Fonte: elaborado pelos autores, 2021.

Para o cálculo do volume utilizado na irrigação foi utilizada a média aritmética da área da borda superior e inferior do vaso. O volume de água utilizado foi ajustado a cada 30 dias (ACOSTA, 2009). Nos primeiros 30 dias as plantas receberam $100 \mathrm{~mm}$ de água. De 30-60 dias foram irrigadas com 200 $\mathrm{mm}$ e entre 60-90 dias receberam $300 \mathrm{~mm}$, dessa forma, foram irrigadas com a quantidade de $600 \mathrm{~mm}$ para todo o ciclo da cultura (90 dias), de acordo com as recomendações de Cavalcante Junior et al. (2013).

Ao aparecer o primeiro botão floral, as laterais da casa de vegetação foram abertas e uma colmeia de abelhas Apis mellifera foi alocada a uma distância de $10 \mathrm{~m}$. Para avaliar a influência da polinização no estágio R4 foram colocadas toucas (toucas de cozinheiro) descartáveis de TNT (polipropileno), para cobrir os capítulos (inflorescência); estas foram colocadas de maneira intercalada, a fim de evitar o efeito da localização. Sendo assim, das 26 plantas adubadas, 13 foram expostas à polinização e 13 (com a touca) não foram polinizadas bioticamente, e o mesmo procedimento foi efetuado para as plantas que não receberam adubo. 
Para verificar a visitação das abelhas nas inflorescências durante todo o período de florescimento, cada uma das plantas (sem touca) foi observada durante 01 minuto a cada hora, das 07:00 às 18:00 horas por um período de 25 dias (período entre a $1^{\text {a }}$ planta entrar em estágio R5 e a última encerrar o estágio R6). Para verificar o efeito do horário do dia na abundância de polinizadores foram selecionados oito dias com temperatura, umidade e pluviosidade semelhantes, utilizando termômetro de bulbo seco, bulbo úmido e observação, respectivamente.

Após a maturação dos capítulos, estes foram colhidos, secados em estufa de circulação forçada com temperatura de $55^{\circ} \mathrm{C}$ por 72 horas. Foi analisado o número de aquênios por inflorescência, peso dos aquênios por inflorescência e produção total. Os aquênios presentes nos capítulos foram retirados e beneficiados no Laboratório de Análise de Alimentos e Nutrição Animal (LANA) pertencente à Universidade do Estado de Mato Grosso (UNEMAT). A estimativa da produção em kg ha- ${ }^{1}$ foi realizada a partir da recomendação de 50 mil plantas por hectare (IAC, 2019). Não foi usada nenhuma classe de agrotóxicos.

Utilizou-se um delineamento inteiramente casualizado em arranjo fatorial 2x2 (adubado e não adubado e polinizado e não polinizado). Os dados de produção apresentaram normalidade pelo teste de Shapiro-Wilk e homogeneidade das variâncias pelo teste de Bartlett e, posteriormente, foram submetidos à análise de variância ao nível de $5 \%$ de probabilidade. Com a finalidade de verificar se houve diferença na abundância de visitantes florais em girassol entre o início e o final do período de florescimento e nos diferentes horários do dia foi empregado o teste não paramétrico de Kruskall-Wallis, pois estes dados não atenderam aos pressupostos de normalidade e homogeneidade de variâncias de acordo com os testes de Shapiro-Wilk e Bartlett, respectivamente. Foi utilizado o software estatístico R, versão 3.6.3, para as análises.

\section{RESULTADOS E DISCUSSÃO}

Para os visitantes florais foram observados 966 indivíduos pertencentes à Classe Insecta. Houve um predomínio da ordem Hymenoptera, em que foi registrado um total de 540 abelhas de espécies nativas e 361 indivíduos da espécie Apis mellifera, e ainda outros 65 visitantes (Outros) (Figura 1a), pertencentes à ordem Lepidoptera, Coleoptera, Diptera, Orthoptera e Hemiptera.

Houve presença de polinizadores durante todo o período do dia avaliado, no entanto, foi possível observar diferença na abundância de visitantes florais entre os diferentes horários do dia (Figura 1b), resultado estatisticamente significativo pelo teste de Kruskall-Wallis (Qui-quadrado=137,04, g.l.=11, p=2,2e-16). Entre 
a abertura e o amadurecimento das flores não houve variações significativas na abundância de polinizadores, demonstrando que há presença de polinizadores durante todo o período de florescimento (Figura 1c), resultado não significativo a 5\% de probabilidade pelo teste de Kruskall-Wallis (Qui-quadrado= 24, g.l.=24, p=0,46).

Figura 1a - Diagrama de Whitakker demonstrando a abundância total observada dos diferentes visitantes florais em girassol

Figura 1b - Abundância de visitantes florais entre os diferentes horários do dia. Gráfico de caixas indicando a mediana (linha sólida no interior da caixa), média (ponto cinza), e dispersão (quartis inferior, superior e outliers). As diferenças foram significativas pelo teste de Kruskall-Wallis (qui-quadrado=137.04, g.l.=11, $\mathrm{p}=2,2 \mathrm{e}-16)$
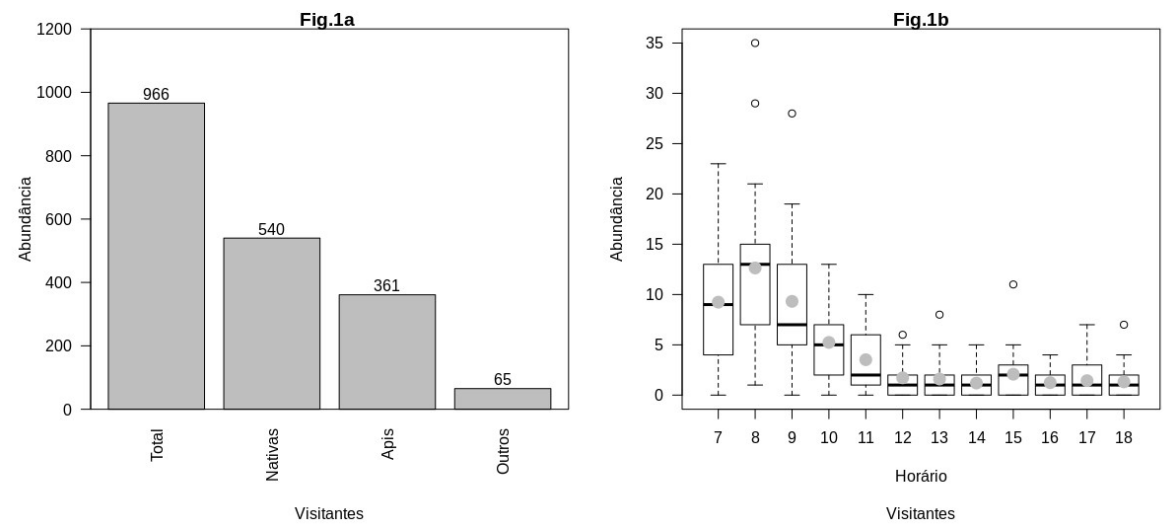

Fonte: elaborado pelos autores, 2021.

Figura 1c - Número médio de visitantes florais observados em cada capítulo por dia, resultado não significativo a 5\% de probabilidade pelo teste de KruskallWallis (Qui-quadrado $=24$, g.1. $=24, \mathrm{p}=0,46$ )

Fig.1c

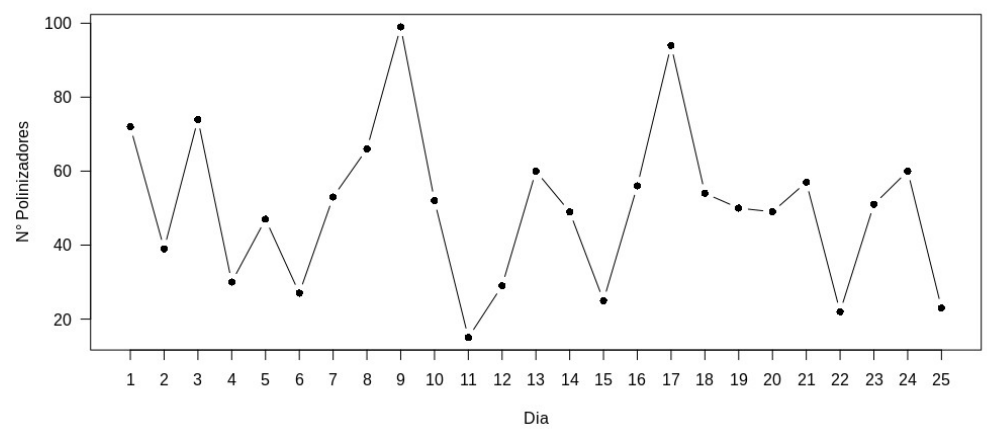

Fonte: elaborado pelos autores, 2021. 
A maior abundância de visitantes florais foi observada no período da manhã, corroborando com os dados observados por (TEIXEIRA; ZAMPIERON, 2008; ROLIM et al., 2011). Provavelmente isto se trata de uma questão de eficiência com o consumo mínimo de energia para o máximo de coleta, pois, segundo Gomes et al. (2012), a coleta de pólen é realizada no período da manhã e o néctar é coletado ao longo de todo o dia, ou seja, no período da manhã há presença de pólen e néctar.

No período da manhã (até as 11:00h) foram observados 73,5\% dos visitantes, e apesar da baixa abundância após às 11:00h, estes representam 26,5\% do número do total. Sendo assim não é recomendável o uso de produtos nocivos aos polinizadores em nenhum horário do dia, pois levaria à morte de muitos polinizadores e, ainda, dependendo do IA utilizado, os poucos polinizadores que entram e contato poderiam não morrer na lavoura, mas ao entrar na colmeia contaminariam vários outros, levando ao extermínio da colônia.

O mesmo se aplica para pulverização durante qualquer período de florescimento do girassol, uma vez que não houve variações na abundância de polinizadores durante todo o período de florescimento. Pois, segundo Oliveira et al. (2018), o uso de agrotóxicos diminui a abundância de polinizadores e a produtividade do girassol. Principalmente porque já é comprovado que todas as classes de agrotóxicos (inseticida, herbicida e fungicida) são prejudiciais às abelhas (ABRAMSON et al., 2004; BOILY et al., 2013; DEGRANDI-HOFFMAN et al., 2015; IUPAC, 2018; KIDD; JAMES, 1991; LEE et al., 2008; LEITE et al., 2015; MARTINELLO et al., 2017; MOGREN; LUNDGREN, 2016; SCHRICKER; STEPHEN, 1970; SOUSA et al., 2013; VIJAYKUMAR; SHIVSHANKAR, 2017).

Assim como observado por Teixeira e Zampieron (2008), Mallinger e Prasifka (2017a), Mallinger e Prasifka (2017b) e Silva et al. (2018), a espécie Apis mellifera foi a mais abundante, entretanto ao somar as espécies nativas e comparálas com a Apis mellifera as nativas foram encontradas em maior número, ainda deve-se esclarecer que para este experimento uma colmeia de Apis mellifera foi alocada próximo ao plantio, e as abelhas nativas nidificavam nas proximidades ( 2 reservas, 1 a 30 e outra a $150 \mathrm{~m}$ de distância do local de plantio).

A adubação foi o fator que mais contribuiu para o aumento da produção de girassol, com o incremento médio de 308,64\%. Adicionalmente, a polinização contribuiu com o aumento de 13,4\% para as plantas não adubadas e em 25,8\% para as que foram adubadas (Figura 2). 
Figura 2 - Médias (pontos) e erro padrão (barras) da produção de sementes de girassol em kg ha- ${ }^{1}$ para os tratamentos não adubado e não polinizado (A), não adubado e polinizado (B), adubado e não polinizado (C) e adubado e polinizado (D). As diferenças foram estatisticamente significativas a $5 \%$ de probabilidade ANOVA $\left(\mathrm{F}=64,29\right.$, g.l. $\left.=51, \mathrm{p}=2-^{16}\right)$

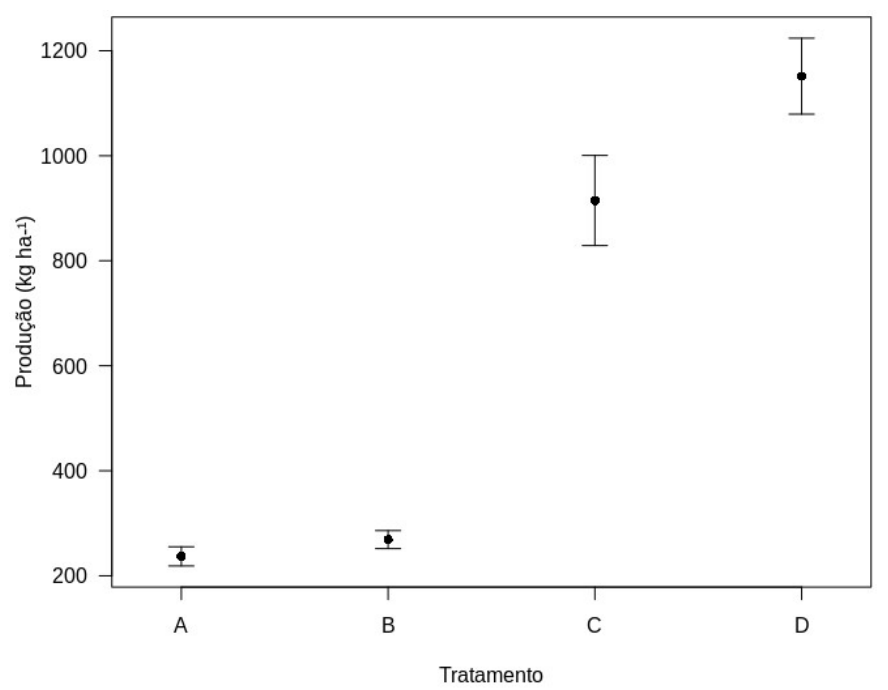

Fonte: elaborado pelos autores, 2021.

Para as plantas não adubadas não houve incremento produtivo com o uso da polinização, corroborando com os dados observados por Tamburini et al. (2015), pois segundo os autores não há efeito da polinização para solos de baixa fertilidade. Os dois principais fatores estudados que influenciaram no aumento da produtividade é o número de grãos e o peso deles.

A polinização foi responsável principalmente pelo aumento no número de grãos (NG), ou seja, este é um fator associado à melhoria na eficiência reprodutiva da planta, enquanto a adubação está mais relacionada à capacidade da planta em aumentar o peso do grão (PG), por isso nas plantas que não foram adubadas não houve diferença na produção observada contrapondo ao verificado para as plantas adubadas.

Outro possível fator que contribuiu para o incremento produtivo seria o aumento da taxa de visitação nas plantas adubadas, pois, segundo Malliger e Prasifka, (2017b), a taxa de visitação aumenta com o incremento da produção de néctar pelas plantas, e é sabido que plantas bem nutridas produzem maior quantidade de néctar e/ou com maior concentração de açúcares. Resultados estes que corroboram com Tamburini et al. (2015), pois, segundo estes, para haver 
efeito da fecundação auxiliada por animais sobre o incremento produtivo do girassol é importante que a planta tenha provimentos nutricionais adequados.

Para o cultivo de girassol SYN 039A há aumento da produtividade com o uso da polinização biótica. O mesmo foi observado por Chambó et al. (2010), pois verificaram aumento produtivo para as cultivares Multissol, M734, Catissol 01, Aguará e Embrapa 122, no entanto, os autores não observaram incremento produtivo com os híbridos Charrua, MG2, Aguará e Helio 360, assim como o encontrado por Mallinger e Prasifka, (2017a), que verificaram efeito para algumas cultivares e não para outros.

Moreti et al. (1996) também encontraram diferença significativa para a cultivar Anhandy. Bartual, Marini e Moonen (2018) observaram aumento produtivo mesmo para as cultivares de girassol desenvolvidas para realizar a autopolinização. Ainda Nderitu et al. (2008) verificaram incremento produtivo de $53 \%$ para a cv, hybrida 8998 , correspondendo ao aumento da receita pela venda da produção em 51\%.

Para as plantas que não receberam adubo, mas foram polinizadas houve elevação do NG em 49\% enquanto o peso PG reduziu 41\%. Isto está associado ao aumento da eficiência reprodutiva oferecida pela polinização biótica e a falta de nutrientes para encher todos os grãos, pois para as plantas adubadas a polinização incrementou 15,6\% o NG e 3,44\% o PG (Tabela 2).

Tabela 2 - Número de grãos por capítulo (NG), peso médio do grão (PG) e a produção estimada para 50 mil plantas por hectare e seus respectivos coeficientes de variação $\left(\mathrm{CV}^{\%} \%\right)$

\begin{tabular}{l|c|c|c}
\hline Tratamentos & NG & PG $(\mathrm{g})$ & ${\text { Produção }\left(\mathrm{kg} \mathrm{ha-}{ }^{-}\right)}$ \\
\hline Não polinizado e não adubado & $156,14(43,3)$ & $0,0304(54,4)$ & $237,01(27,8)$ \\
\hline Polinizado e não adubado & $232,78(14,2)$ & $0,0231(24,1)$ & $268,94(22,9)$ \\
\hline Não polinizado e adubado & $355,57(19,4)$ & $0,058(45,2)$ & $914,75(33,7)$ \\
\hline Polinizado e adubado & $411,21(10,1)$ & $0,060(24,3)$ & $1151,50(22,6)$ \\
\hline CV\% & 39,95 & 52,17 & 70,56 \\
\hline
\end{tabular}

Fonte: elaborado pelos autores, 2021.

O método de cobertura dos capítulos de girassol utilizado no presente experimento foi eficiente para evitar o acesso dos visitantes florais nas inflorescências. De acordo com Moreti et al. (1996), houve aumento no número e no peso dos grãos para as plantas que foram polinizadas, porém estes não observaram diferença estatística entre as plantas em que seus capítulos foram ensacados com filó e os sem cobertura alguma, os autores não relataram a causa 
do ocorrido, mas concluem que este não é um método adequado para impedir as abelhas de polinizar o girassol, e recomendam o uso de gaiolas para impedir a visitação. Observou-se que algumas espécies de abelhas nativas possuem a capacidade de transpassar obstáculos com maior facilidade do que a Apis mellifera, portanto qualquer pequeno espaço entre a haste de capítulo e o tecido poderá ser transpassado por estas espécies, assim ocorrendo a polinização.

Silva et al. (2018) também observaram que a polinização aumenta o peso dos grãos. Adicionalmente, Perrot et al. (2018) indicam o uso de colmeias no interior das plantações de girassol para incrementar significativamente a produção de girassol. Isto pode ser aplicado principalmente para áreas de plantio muito extensas onde é impossível garantir a presença de polinizadores e/ou em áreas em que a população de abelhas já diminuiu drasticamente.

Ainda conforme pode ser observado (Tabela 2), a polinização contribuiu para maior padronização das características analisadas (menores coeficientes de variação). Esta característica é importante para o sistema produtivo, principalmente para o setor de produção de sementes, pois ao se diminuir as variações de peso dos grãos e número de grãos (que é uma característica relacionada ao tamanho do grão), consequentemente se diminuem as perdas no processo de colheita, e também os custos principalmente com a seleção de sementes para o plantio, uma vez que para esta finalidade as sementes devem apresentar uniformidade para garantir a melhor homogeneidade de distribuição e profundidade de semeadura.

Segundo Silva et al. (2010), a classificação por tamanho ou peso das sementes garante a uniformização da emergência das plântulas e a obtenção de mudas de tamanho semelhante ou de maior vigor. Ainda conforme relatado por Nunes et al. (2016), sementes maiores ou mais pesadas apresentam melhor taxa de germinação e isso pode ser explicado pelo fato de que estas acumularam maior quantidade de fotoassimilados durante sua formação, possuindo embriões bem formados e com maior quantidade de reservas, sendo assim, mais vigorosas do que as de menor tamanho.

De acordo com os dados de Silva et al. (2018), o desvio padrão do peso de 100 sementes de girassol foi menor para as plantas polinizadas $(7,35)$ do que para as plantas não polinizadas (10,27). Segundo Nobre et al. (2015), houve desuniformidade na massa das sementes, possivelmente são genótipos que possuem alta taxa de dependência de polinizadores e/ou o uso de polinizadores poderia auxiliar na melhoria deste parâmetro.

A cultivar SYN 039A, recebendo teores nutricionais adequados responde a polinização biótica com $25,8 \%$ de aumento de produtividade, sabendo que os produtores realizam adubação e/ou correção do solo, qualquer aplicação de 
agrotóxicos só se justificaria se a perda de produção pelo ataque de pragas fosse superior a $25 \%$ (sem incluir os custos com a compra e aplicação destes insumos), importante esclarecer ainda que existem outros custos diretos e indiretos associados às pessoas que manipulam estas substâncias, pois há riscos relacionados a problemas de saúde como alergias, lesões hepáticas, neoplasias, doenças de ordem neurotóxica, depressão, suicídio, câncer, dentre outras (BOTELHO et al., 2017; CHRISMAN et al., 2009; CONTI et al., 2018; KIM; KABIR; JAHAN, 2016; LOVISSI et al., 2009; MACHADO; SANTOS, 2015; MREMA et al., 2017; PERTILE et al., 2018; SHINOHARA et al., 2017; STALLONES; BESELER, 2002; TOMIAZZI et al., 2018; VALKE et al., 2017).

\section{CONCLUSÃO}

Há incremento produtivo quando há polinização biótica em plantas de girassol SYN 039A, mas este efeito é dependente do conteúdo de nutrientes disponíveis para a planta. Há presença de polinizadores durante o período de florescimento e em qualquer horário do dia, desta maneira só é viável economicamente o uso de agrotóxicos se as perdas de produção ocasionada pelas pragas forem superiores a $25 \%$.

\section{REFERÊNCIAS}

ABRAMSON, C. I.; SQUIRE, J.; SHERIDAN, A.; MULDER JUNIOR, F.G. The effect of insecticides considered harmless to honey bees (Apis mellifera): proboscis conditioning studies by using the insect growth regulators Tebufenozide and Diflubenzuron. Environmental Entomology, [s. l.], v. 33, n. 2, p. 378-388, 2004.

\section{ACOSTA, J. F. Consumo hídrico da cultura do girassol irrigada na} região da chapada do Apodi - RN. 2009. 73 f. Dissertação (Mestrado em Meteorologia) - Centro de Tecnologia e Recursos Naturais, Universidade Federal de Campina Grande, Campina Grande, 2009.

ALVAREZ V. H.; DIAS, L. D; LEITE, P. B.; SOUZA, R. B.; RIBEIRO JUNIOR, E. S. Poda de raízes e adubação para crescimento do cafeeiro cultivado em colunas de solo. Revista Brasileira de Ciência do Solo, [s. l.], v. 30, p. 111-119, 2006.

ARENA, M.; SGOLASTRA, F. A meta-analysis comparing the sensitivity of bees to pesticides. Ecotoxicology, [s. l.], v. 23, p. 324-334, 2014. 
BARTUAL, A. M.; MARINI, S. B. G.; MOONEN, A. C. Local and landscape factors affect sunflower pollination in a Mediterranean agroecosystem. Plos One. [s. l.], v. 13, n. 9, p. 1-18, 2018.

BOILY, M.; SARRASIN, B.; DEBLOIS, C.; ARAS, P.; CHAGNON, M. Acetylcholinesterase in honey bees (Apis mellifera) exposed to neonicotinoids, atrazine and glyphosate: laboratory and field experiments. Environmental Science and Pollution Research, [s. l.], v. 20, p. 5603-5614, 2013.

BOMMARCO, R.; KLEIJN, D.; POT'TS, S. G. Ecological intensification: Harnessing ecosystem services for food security. Trends in Ecology and Evolution, [s. l.], v. 28, n. 4, p. 230-238, 2013.

BOTELHO, P. C. S.; ROCHA, V. G.; DUTRA, T. R.; FEITOSA, J. C. A.; ASSIS, R. C.; MIGUEL, T. S.; ANJOS, N. K. C.; COSTA, D. A.; LOPES, L. C. P.; ROCHA, L. L. V. Auditory Health and Risk Factors in Rural Workers Exposed to Pesticides in Piedade de Caratinga County-Minas Gerais. Journal of Biosciences and Medicines, [s. l.], v. 5, p. 37-55, 2017.

CAVALCANTE JUNIOR, E. G.; MEDEIROS, J. F.; MELO, T. K.; SOBRINHO, J. E.; BRISTOT, G.; ALMEIDA, B. M. Necessidade hídrica da cultura do girassol irrigado na chapada do Apodi. Revista Brasileira de Engenharia Agrícola e Ambiental, Campina Grande, v. 17, n. 3, p. 261-267, 2013.

CHAMBÓ. E. D.; GARCIA, R. C.; OLIVEIRA, N. T. E.; CHIRÉA, A. Produção de genótipos de girassol pela ação de insetos polinizadores. Revista Varia Scientia, [s. l.], v. 9, n. 15, p. 131-139, 2010.

CHRISMAN, J. R.; KOIFMAN, S.; SARCINELLI, P. N.; MOREIRA, J. C.; KOIFMAN, R. J.; MEYER, A. Pesticide sales and adult male cancer mortality in Brazil. International Journal of Hygiene and Environmental health, [s. l.], v. 212, p. 310-321, 2009.

CLARK, M.; TILMAN, D. Comparative analysis of environmental impacts of agricultural production systems, agricultural input efficiency, and food choice. Enviromental Research Letters, [s. l.], v. 12, n. 6, p. 1-11, 2017.

CONTI, C. L.; BARBOSA, W. M.; SIMÃO, J. B. P.; ÁLVARES-DA-SILVA, A. M. Pesticide exposure, tobacco use, poor self-perceived health and presence of chronic disease are determinants of depressive symptoms among coffee growers from Southeast Brazil. Psychiatry Research, [s. l.], v. 260, p. 187-192, 2018. 
DEGRANDI-HOFFMAN, G.; CHEN, Y.; DEJONG, E. W.; CHAMBERS, M. L.; HIDALGO, G. Effects of oral exposure to fungicides on honey bee nutrition and virus levels. Journal of Economic Entomology, [s. l.], v. 251, p. 2518-2528, 2015.

GALLAI, N.; VAISSIÈRE, B.E. Global action on pollination services for sustainable agriculture: guidelines for the economic valuation of pollination services at a national scale. Food and Agriculture Organization, Rome, FAO, 2009, 11 p.

GOMES, G. et al. Frequência de visitas de abelhas (Apis mellifera) em plantas de gergelim (Sesamum indicum) e girassol (Helianthus annuus). In: CONGRESSO NORTE E NORDESTE DE PESQUISA E INOVAÇÃO, 7. 2012, Palmas. Anais [...]. Palmas: IFTO, 2012. p. 1-6.

GREENLEAF, S. S.; KREMEN, C. Wild bees enhance honey bees' pollination of hybrid sunflower. Procedings of the Nationa Academy of Science (PNAS), [s. l.], v. 103, n. 37, p. 13890-13895, 2006.

IAC. Girassol. Instituto Agronomico de Campinas, Campinas, 2019. Disponível em: http://www.iac.sp.gov.br/cultivares/inicio/folders/girassol/ IACUruguai.htm. Acesso em: 05 fev. 2020.

IUPAC. Carbendazim (Ref: BAS 346F). International Union of Pure and Applied Chemistry, [s. l.], 2018. Disponível em: http:/ / sitem.herts.ac.uk/ aeru/iupac/Reports/116.htm. Acesso em: 01 set. 2018.

JUNG, A. H. Impacto de inseticidas aplicados em soja sobre abelhas melíferas. 2014. 63 f. Dissertação (Mestrado em Engenharia Agrícola) Centro de Ciências Rurais, Programa de Pós-Graduação em Engenharia Agrícola, Universidade Federal de Santa Maria, Santa Maria, 2014.

KIDD, H.; JAMES, D. R. The agrochemicals handbook. 3. ed. Surrey, England: Royal Society of Chemistry Information Systems, 1991. 313 p.

KIM, K.; KABIR, E.; JAHAN, S.A. Exposure to pesticides and the associated human health effects. Science of the Total Environment, v.575, p.525-535, 2016. KLEIN, A. M.; VAISSIÈRE, B. E.; CANE, J. H.; STEFFAN-DEWENTER, I.; CUNNINGHAM, S. A.; KREMEN, C.; TSCHARNTKE, T. Importance of pollinators in changing landscapes for world crops. Proceedings of the Royal Society, Biological Sciences, [s. l.], v. 274, n. 1608, p. 303-313,2007. 
KÖPPEN, W. Die klimate der Erde. Berlin: W. Guyter, 1923. 369p.

LEE, M.; CHOI, K.; LEE, S.; KIM, J. Physical symptoms of honey bees, Apis mellifera L., after contact with residual acephate on apple trees. Korean Journal of Apiculture, [s. l.], v. 23, n. 4, p. 235-239, 2008.

LEITE, G. L. D.; PAULO, P. D.; ZANUNCIO, J. C.; ALVARENGA, A. C.; SOARES, M. A.; TAVARES, W. S.; TUFFI-SANTOS, L. D.; SPÍNOLAFILHO, P. R. C. Effects of atrazine-based herbicide on emergence and sex ratio of Trichogrammatidae (Hymenoptera). Florida Entomologist, [s. l.], v. 8, n. 3, p. 899-902, 2015.

LOVISI, G. M.; SANTOS, S. A.; LEGAY, L.; ABELHA, L.; VALENCIA, E. Análise epidemiológica do suicídio no Brasil entre 1980 e 2006. Revista Brasileira de Psiquiatria, [s. l.], v. 31, p. 86-93, 2009.

MACHADO, D. B.; SANTOS, D. N. Suicídio no Brasil, de 2000 a 2012. Jornal Brasileiro de Psiquiatria, Rio de Janeiro, v. 64, n. 1, p. 45-54, 2015.

MALLINGER, R.; PRASIFKA, J. Benefits of insect pollination to confection sunflowers differ across plant genotypes. Crop Science, [s. l.], v. 57, p. 3264-3272, 2017a.

MALLINGER, R.; PRASIFKA, J. Bee visitation rates to cultivated sunflowers increase with the amount and accessibility of nectar sugars. Jounal of Applied Entomology, [s. l.], v. 141, n. 7, p. 561-573, 2017b.

MALLINGER, R.; BRADSHAW, J.; VARENHORST, A. J.; PRASIFKA, J. R. Native solitary bees provide economically significant pollination services to confection sunflowers (Helianthus annuus L.) (asterales: asteraceae) grown across the northern great plains. Journal of Economic Entomology, [s. l.], v. 112, n. 1, p. 40-48, 2019.

MARTINELLO, M.; BARATTO, C.; MANZINELLO, C.; PIVA, E.; BORIN, A.; TOSON, M.; GRANATO, A.; BONIOTTI, M.B.; GALLINA, A.; MUTINELLI, F. Spring mortality in honey bees in northeastern Italy: detection of pesticides and viruses in dead honey bees and other matrices. Journal of Apicultural Research, [s. l.], v. 56, n. 3 p. 1-16, 2017.

MOGREN, C. L.; LUNDGREN, J. G. Neonicotinoid-contaminated pollinator strips adjacent to cropland reduce honey bee nutritional status. Nature, California, v. 6, n. 29608, p. 1-10, 2016. 
MORETI, A. C. C. C.; SILVA, R. M. B.; SILVA, E. C. A.; ALVES, M. L. T. M. F.; OTSUK, I. P. Aumento na produção de sementes de girassol (Helianthus annuus) pela ação de insetos polinizadores. Scientia Agricola, São Paulo, v. 53, n. 2, p. 1-4, 1996.

MREMA, E. J.; NGOWI, A. V.; KISHINHI, S. S.; MAMUYA, S. H. Pesticide exposure and health problems among female horticulture workers in Tanzania. Environmental Health Insights, [s. l.], v. 11, p. 1-13, 2017.

NDERITU J.; NYAMASYO, G.; KASINA, M.; ORONJE, M. L. Diversity of sunflower pollinators and their effect on seed yield in Makueni District, Eastern Kenya. Spanish Journal of Agricultural Research, [s. l.], v. 6, n. 2, p. 271-278, 2008.

NOBRE, D. A. C.; COSTA, C. A.; BRANDÃO JUNIOR, D. S.; RESENDE, J. C. F.; FLÁVIO, N. S. D. Qualidade das sementes de girassol de diferentes genótipos. Ciência Rural, Santa Maria, v. 45, n. 10, p. 1729-1735, 2015.

NUNES, R. T. C.; CANGUSSU, A. C. V.; OLIVEIRA, C. C.; SANTOS, A. P. S.; SOUZA, U. O.; MORAIS, O. G. Efeito da classificação por tamanho em sementes de girassol na avaliação da qualidade fisiológica. Cultura

Agronômica, Ilha Solteira, v. 25, n. 1, p. 105-115, 2016.

OLIVEIRA, A. C.; JUNQUEIRA, C. N.; AUGUSTO, S. C. Pesticides affect pollinator abundance and productivity of sunflower (Helianthus annuus L.). Journal of Apicultural Research, [s. l.], v. 58, n. 1, p. 2-8, 2018.

PERROT, T.; GABA, S.; RONCORONI, S.; GAUTIER, J-L.; SAINTILAN A.; BRETAGNOLLE, V. Experimental quantification of insect pollination on sunflower yield, reconciling plant and field scale estimates. Basic and Applaied Ecology, [s. l.], v. 34, p. 75-84, 2018.

PERTILE, E.; MATIAS, M. I.; RIBEIRO, Z. S.; POETA, J.; RONCADA, C. Evidências experimentais e epidemiológicas entre exposição aos agrotóxicos e o desenvolvimento de câncer de mama. Revista Brasileira de Pesquisa em Saúde, Vitória, v. 20, n. 1, p. 137-147, 2018.

PHALAN, B.; GREEN, R. E.; DICKS, L. V.; DOTTA, G.; FENIUK, C.; LAMB, A.; STRASSBURG, B. B. N.; WILLIAMS, D. R.; ZU ERMGASSEN, E. K. H. J.; BALMFORD, A. How can higher-yield farming help to spare nature? Science, New York, v. 315, n. 6272. p. 450-451, 2016. 
ROLIM, G. G; ANDRADE, W. C.; PEREIRA, G. A.; SANTOS. L. G.; ANDERLEY, E. A. Frequência de visitas de abelhas (Apis mellifera) em diferentes cultivares de girassol (Helianthus annuus L.) no semiárido paraibano. Enciclopédia Biosfera, Jandaia, v. 7, n. 12, p. 1-6, 2011.

SCHRICKER, B.; STEPHEN, W. P. The effect of sublethal doses of parathion on honeybee behaviour. I. Oral administration and the communication dance. Journal of Apicultural Research, [s. l.], v. 9, n. 3, p. 141-153, 1970.

SHINOHARA, N. K. S.; PADILHA, M. R. F.; OLIVEIRA, F. H. P. C.; CABRAL, J. V. B. Insegurança alimentar no uso indiscriminado de agrotóxicos. Revista Higiene Alimentar, São Paulo, v. 31, n. 266/267, p. 17-21, 2017. SILVA, C. A. S.; GODOY, W. A. C.; JACOB, C. R. O.; THOMAS, G.; CÂMARA, G. M. S.; ALVES, D. A. Bee pollination highly improves oil quality in sunflower. Sociobiology, [s. l.], v. 65, n. 4, p. 583-590, 2018.

SILVA, K. S.; MENDONÇA, V.; MEDEIROS, L. F.; FREITAS, P. S. C.; GÓIS, G. B. Influência do tamanho da semente na germinação e vigor de mudas de jaqueira (Artocarpus heterophyllus Lam.). Revista Verde de Agroecologia e Desenvolvimento Sustentável, Pombal, v. 5, n. 4, p. 217-221, 2010.

SILVEIRA, R. K.; GALBIATI, C. Crescimento no uso de agrotóxicos e sua relação com polinizadores, saúde humana e economia. In: SGUAREZI, S. B. (org.). Ambiente e sociedade no Brasil central: diálogos interdisciplinares e desenvolvimento regional. Cáceres: Oikos Editora, 2019. p. 183-197.

SOUSA, J. R. L.; AMARANTE JUNIOR, O. P.; BRITO, N. M.; FRANCO, T. C. R. S. Ação de pesticidas sobre abelhas: avaliação do risco de contaminação de méis. Acta Tecnológica, São Luís, v. 8, n. 1, p. 28-36, 2013.

STALLONES, L.; BESELER, C. Pesticide poisoning and depressive symptoms among farm residents. Annals of Epidemiology, [s. l.], v. 12, n. 6, p. 389-394, 2002.

TAMBURINI, G.; BERTI, A.; MORARI, F.; MARINI, L. Degradation of soil fertility can cancel pollination benefits in sunflower. Oecology, [s. l.], v. 180, n. 2, p. 581-587, 2015.

TEIXEIRA, L. M. R.; ZAMPIERON, S. L. M. Estudo da fenologia, biologia floral do girassol (Helianthus annuus, Compositae) e visitantes florais associados, em diferentes estações do ano. Ciência et Praxis, Belo Horizonte, v. 1, n. 1,p. 5-14, 2008. 
TOMIAZZI, J. S.; JUDAI, M. A.; NAI, G. A.; PEREIRA, D. R.; ANTUNES, P. A.; FAVARETO, A. P. A. Evaluation of genotoxic effects in Brazilian agricultural workers exposed to pesticides and cigarette smoke using machinelearning algorithms. Environmental Science and Pollution Research, [s. l.], v. 25, p. 1259-1269, 2018.

VALCKE, M.; BOURGAULT M-H.; ROCHETTE, L.; NORMANDIN, L.; SAMUEL, O.; BELLEVILLE, D.; BLANCHET, C.; PHANEUF, D. Human health risk assessment on the consumption of fruits and vegetables containing residual pesticides: A cancer and non-cancer risk/benefit perspective.

Environment International, [s. l.], v. 108, p. 63-74, 2017.

VALDOVINOS-NÚÑEZ, G. R.; QUEZADA-EUÁN, J. J. G.; ANCONAXIU, P.; MOO-VALLE, H.; CARMONA, A.; SÁNCHEZ, E. R. Comparative toxicity of pesticides to stingless bees (Hymenoptera: Apidae: Meliponini). Journal of Economic Entomology, [s. l.], v. 102, n. 5, p. 1737-1742, 2009.

VIJAYKUMAR, P. V.; SHIVSHANKAR, P. C. Monitoring on impact of insecticides on mortality of honey bees (Apis mellifera L.) in front of beehives. Journal of Applied and Natural Science, [s. l.], v. 9, n. 2, p. 905-911, 2017. 
